\title{
Valor de uso medicinal de la flora empleada por el poblador de Victor Larco, Trujillo, La Libertad, Perú
}

\author{
Value of medicinal use of the flora used by the people of Victor \\ Larco, Trujillo, La Libertad, Peru
}

\author{
José Mostacero-León ${ }^{1} \square$, (D) Kevin Zavaleta Villacorta ${ }^{1}$, (D) Luis Taramona-Ruíz ${ }^{2}$, \\ Anthony De La Cruz Castillore, (iD Efraín Gil Rivero ${ }^{1}$
}

${ }^{1}$ Universidad Nacional de Trujillo. Trujillo, Perú

${ }^{2}$ Universidad Le Cordon Bleu. Lima, Perú

Recibido: 10/04/2020_ Revisado: 20/05/2020Ａceptado: 20/09/2020Ｐublicado: 16/12/2020

\section{RESUMEN}

La expansión demográfica a nivel mundial, y en el Perú, particularmente trae consigo un sin número de problemas, destacando sobre ellos, los relacionados a la salud; siendo este, un pilar fundamental para el progreso y desarrollo de los pueblos; por ello, la presente investigación se avocó a determinar el valor de uso medicinal de la flora empleada por el poblador del distrito de Víctor Larco, Trujillo, La Libertad, Perú. Se aplicaron un total de 96 entrevistas semi - estructuradas a los pobladores del distrito en mención, a fin de obtener información respecto al nombre común, parte utilizada, tipo de preparación, modo de aplicación, usos medicinales, así como el índice de valor de uso (IVU). Paralelamente se colectó, secó, montó, etiquetó y determinó taxonómicamente los especímenes referidos por la población. Se reportan 50 especies de plantas de uso etnomedicinal en el distrito; distribuidas en 46 géneros y 30 familias, de las que destacan por su número de especies: Asteraceae (7), Lamiaceae (4), Fabaceae (3), Liliaceae (3) y Rutaceae (3). Se concluye que las especies más importantes empleadas en el tratamiento y/o cura de las enfermedades padecidas por el poblador del distrito de Victor Larco, según su Indice de Valor de Uso (IVU) son: Linum usitatissimum L. "linaza", Cymbopogon citratus (DC.) Stapf "hierba luisa", Citrus limon (L.) Osbeck "limón”, Aloe vera (L.) Burm.f. "sábila", Solanum tuberosum L. "papa", Hordeum vulgare L. "cebada" y Morinda citrifolia L. "noni". Palabras clave: Valor de uso medicinal, Flora, Víctor Larco, Trujillo.

\section{ABSTRACT}

The demographic expansion worldwide, and in Peru, particularly brings with it a number of problems, highlighting among them, those related to health; being this, a fundamental pillar for the progress and development of the peoples; for this reason, the present investigation focused on determining the medicinal use value of the flora used by the inhabitants of the Víctor Larco district, Trujillo, La Libertad, Peru. A total of 96 semi-structured interviews were applied to 
the residents of the mentioned district, in order to obtain information regarding the common name, part used, type of preparation, method of application, medicinal uses, as well as the index of use value (IVU). In parallel, the specimens reported by the population were collected, dried, assembled, labeled and taxonomically determined. 50 species of plants for ethnomedical use in the district are reported; Distributed in 46 genera and 30 families, of which they stand out for their number of species: Asteraceae (7), Lamiaceae (4), Fabaceae (3), Liliaceae (3) and Rutaceae (3). It is concluded that the most important species used in the treatment and / or cure of the diseases suffered by the population of the Victor Larco district, according to their Use Value Index (IVU) are: Linum usitatissimum L. "linaza", Cymbopogon citratus (DC.) Stapf "hierba luisa", Citrus limon (L.) Osbeck "limón”, Aloe vera (L.) Burm.f. "sábila", Solanum tuberosum L. "papa", Hordeum vulgare L. "cebada” y Morinda citrifolia L. "noni”. Keywords: Medicinal use value, Flora, Víctor Larco, Trujillo.

\section{INTRODUCCIÓN}

Es irrefutable, negar el hecho que Perú al formar parte de los 12 países megadiversos; alberga una inmensa biodiversidad, entre plantas y animales; recursos que el hombre empíricamente supo aprovechar y así suplir todas sus necesidades, destacando de entre ellas las plantas, y más específicamente la flora medicinal; especies capaces de curar y/o aliviar diferentes enfermedades o dolencias que padeció, padece y padecerá el poblador mundial, en general, y el peruano en particular (Mittermeier et al., 1997; Brack, 2000; Mostacero et al., 2011; Bhat et al., 2016).

Uso etnomedicinal, catalogado hoy en día como "saberes ancestrales"; impartidos en un inicio por los "Hampicamayoc", "Oquetlupuc" y "Sircac", personajes diestros en el arte del correcto y eficaz uso de esta flora medicinal, que los catalogó como médicos del incanato; encargados de transmitir esta herencia ancestral de generación en generación; llegando a nuestros días a perdurar en las comunidades Andino- Amazónicas sobre todo, y más específicamente en la memoria de los curanderos, chamanes y abuelitas (Mostacero et al., 2011; Rengifo et al., 2017).
Legado ancestral, que día a día toma mayor importancia a nivel mundial, esto en suma a que la ciencia y la tecnología avalan cada vez más el uso de las plantas medicinales como alternativa factible y económica en la cura y/o tratamiento de más de 370 enfermedades conocidas hasta la actualidad; al existir plantas que combaten enfermedades que afecten al Sistema: digestivo, al respiratorio, al nervioso, al musculo esquelético, al cardiovascular, al renal, entre otros (Boeing et al., 2012; Cárdenas et al., 2015; Mostacero et al., 2019), a la vez que son capaces de combatir la diabetes, hipercolesterolemia, así como prevenir y enfrentar distintos tipos de cáncer (Boeing et al., 2012; Freedman et al., 2014). Sumado al hecho de que muchas especies retrasan el envejecimiento (Goszcz et al., 2015).

En lo que respecta a Perú y más concretamente en la provincia de Trujillo; en los últimos 10 años se viene generando una notoria expansión demográfica; hecho que conlleva a un aumento desmesurado en la demanda de recursos, bienes y servicios, con la consiguiente sobrexplotación de los mismos y el desmejoro de la calidad de vida de la población más necesitada, dado por 
la aparición de múltiples enfermedades y debido a la escases económica en la que viven; recurriendo por consiguiente al empleo de plantas medicinales como una alternativa de atención primaria para la salud; reflejado en el hecho de que el 72\% de la Población Trujillana las utiliza. (INEI, 2018; De La Cruz y Mostacero, 2019; Rodríguez et al. 2019).

Por lo descrito en los párrafos anteriores y sabiendo que a través de la historia, las elevadas tasas inmigratorias, generalmente estuvieron conformadas por una población rural andina-amazónica de amplio conocimiento etnobotánico; y que con el correr del tiempo se establecieron en las regiones de mayor pujanza económica, siendo la Costa una de ellas y Trujillo la cuarta provincia más poblada del Perú; la presente investigación se avocó a determinar el valor de uso medicinal de la flora empleada por el poblador del distrito de Víctor Larco, Trujillo, La Libertad, Perú; a fin de rescatar este legado ancestral y propalar su conservación, a la par de servir de base para futuras investigaciones del tipo aplicadas que fomenten su aprovechamiento sostenible.

\section{MATERIALES Y MÉTODOS}

\section{Área de estudio}

La presente investigación se desarrolló en el distrito de Víctor Larco, provincia de Trujillo, La Libertad, Perú; ubicado en la parte sur oeste del distrito Trujillo entre los $8^{\circ} 8$ '22.2” de L. S. y $79^{\circ} 2^{\prime} 44.38^{\prime \prime}$ L.O. y a una altitud de 3 m.s.n.m.

\section{Muestra}

La población de estudio estuvo conformada por 96 adultos y adultos mayores de ambos sexos cuyas edades oscilan entre 18 a 72 años de edad, del distrito en mención, que hayan empleado las plantas medicinales y/o preparados a base de ellas, en la cura y/o alivio de sus enfermedades. Dato obtenido, mediante la fórmula propuesta por Bocanegra et al (2011), que considera población finita y varianza desconocida, con un error permisible de $10 \%$ y un nivel de confianza del $95 \%$.

$$
\mathbf{n}=\frac{N Z^{2} p q}{d^{2}(N-1)+Z^{2} p q}
$$

Donde:

n: Tamaño de la muestra; N: Población del estudio ( $\mathrm{n}=68506$ habitantes); p: Proporción de éxito de la característica de interés, igual a $50 \%(0.5)$; q: $1-\mathrm{P}=$ complemento de P; $\alpha / 2$ Z: Coeficiente de confiabilidad al 95\% igual a 1.96; d: Tolerancia de error permisible en la investigación $=(10 \%)$.

\section{Instrumento y Técnicas de recolección de datos}

Para el estudio, se empleó la entrevista semi estructurada; la misma que se aplicó utilizando la técnica "bola de nieve" (Bailey, 1994); en donde, se procedió a entrevistar al primer individuo conocedor de las plantas medicinales, en el distrito de Víctor Larco, quien brindó información requerida acerca de los nombres vulgares, parte utilizada de la planta, tipo de preparación, modo de administración, así como los usos medicinales que les confieren a cada espécimen. Luego, al finalizar la entrevista, se le solicitó, indique el nombre de una persona a quien considere conocedora en el tema; quien luego fue entrevistada y que al igual que la anterior, proporcionó información concerniente a plantas medicinales; y así, a modo de "bola de nieve" se aplicaron los 94 entrevistados restantes. 


\section{Colecta e Identificación de la flora medicinal}

La colecta de los especímenes botánicos, se realizaron paralelamente a la aplicación de las entrevistas. Seguido a la coleta, las plantas fueron transportadas en prensa botánica, al Herbarium Truxillense de la U.N.T (H.U.T.); donde se procedió a secarlos, montarlos, etiquetarlos, preservarlos e identificarlos taxonómicamente. Cabe destacar que también, se hizo uso de claves taxonómicas referidas a la flora peruana, según: Brako y Zarucchi (1993) y Mostacero et al. (2009), agenciados con los portales virtuales: Trópicos y The Plant List.

\section{Análisis de datos}

La información recabada, fue organizada en tablas, con la información encontrada, analizada y contrastada en diferentes tesis, artículos científicos y libros sobre la materia (Mostacero et al., 2011). De igual manera, para la determinación del índice de valor de uso de cada especie (IVU). Este fue estimado se- gún lo propuesto por Zambrano et al. (2015):

$$
I V U=\frac{V U_{i s}}{N_{s}} ;
$$

Donde: $\mathrm{VU}_{\mathrm{is}}$ : Valor de uso de la especie y $\mathrm{N}_{\mathrm{s}}$ : Número de informantes para cada especie.

\section{RESULTADOS Y DISCUSIÓN}

En la Tabla 1. Se detallan los datos referidos a características taxonómicas: familia, nombre científico y común, parte utilizada, tipo de preparación, forma de administración, usos medicinales e índice de valor de uso (IVU); de 50 especies de flora, empleadas medicinalmente por el poblador del distrito de Víctor Larco, distribuidas en 46 géneros y 30 familias (Figura 1); apreciándose a su vez que las Asteraceae, Lamiaceae, Rutaceae, Liliaceae y Fabaceae son las familias más representativas por su número de especies (Figura 2)

Tabla 1. Características taxonómicas y etnomedicinales de la flora empleada por el poblador de Víctor Larco, Trujillo, La Libertad, Perú.

\begin{tabular}{|c|c|c|c|c|c|c|c|c|c|}
\hline $\mathrm{N}$ & FAMILIAS & $\begin{array}{l}\text { NOMBRE } \\
\text { CIENTÍFICO }\end{array}$ & $\begin{array}{l}\text { NOMBRE } \\
\text { COMÚN }\end{array}$ & P.U & T.P & F.A & USOS MEDICINALES & $\mathrm{RU}$ & IVU \\
\hline 1 & ANACARDIACEAE & $\begin{array}{l}\text { Schinus molle } \\
\text { L. }\end{array}$ & "molle" & $\begin{array}{l}\text { Hoja y } \\
\text { corteza }\end{array}$ & $\begin{array}{l}\text { Infusión y } \\
\text { cocimiento }\end{array}$ & Oral & $\begin{array}{l}\text { Acción parasiticida, contra } \\
\text { enfermedades respiratorias, } \\
\text { antidiarreico y depurativa. }\end{array}$ & 22 & 0.23 \\
\hline 2 & APIACEAE & $\begin{array}{l}\text { Coriandrum } \\
\text { sativum L. }\end{array}$ & $\begin{array}{l}\text { "culantro", } \\
\text { "cilantro" }\end{array}$ & Hojas & Infusión & Oral & $\begin{array}{l}\text { Desinfectante intestinal, } \\
\text { sudorificos, propiedades } \\
\text { estomacales y calmar } \\
\text { dolencias de origen } \\
\text { nervioso. }\end{array}$ & 25 & 0.26 \\
\hline 3 & APIACEAE & $\begin{array}{l}\text { Apium } \\
\text { graveolens L. }\end{array}$ & "apio" & Hojas & $\begin{array}{l}\text { Infusión y } \\
\text { cocimiento }\end{array}$ & Oral & $\begin{array}{l}\text { Propiedades contra la } \\
\text { neumonía, el hipo, colitis } \\
\text { crónicas, la artritis y la gota. }\end{array}$ & 6 & 0.06 \\
\hline 4 & ASTERACEAE & $\begin{array}{l}\text { Bidens pilosa } \\
\text { L. }\end{array}$ & "amor seco" & Rama & Cocimiento & baños & $\begin{array}{l}\text { Acción desinflamante, } \\
\text { diarreas, contra dolores de } \\
\text { cabeza, úlceras dérmicas y } \\
\text { conjuntivitis }\end{array}$ & 3 & 0.03 \\
\hline 5 & ASTERACEAE & $\begin{array}{l}\text { Tagetes } \\
\text { pusilla Kunth. }\end{array}$ & $\begin{array}{l}\text { "anis } \\
\text { serrano" }\end{array}$ & $\begin{array}{l}\text { Toda la } \\
\text { planta }\end{array}$ & $\begin{array}{l}\text { Infusión y } \\
\text { cocimiento }\end{array}$ & Oral & $\begin{array}{l}\text { Acción antipirética, cólicos } \\
\text { menstruales, contra } \\
\text { escalofríos nervios, } \\
\text { digestiva y dolor de cabeza. }\end{array}$ & 20 & 0.21 \\
\hline
\end{tabular}




\begin{tabular}{|c|c|c|c|c|c|c|c|c|c|}
\hline 6 & ASTERACEAE & $\begin{array}{l}\text { Matricaria } \\
\text { chamomilla L. }\end{array}$ & $\begin{array}{l}\text { "manzanilla } \\
\text { " }\end{array}$ & $\begin{array}{l}\text { Hoja y } \\
\text { flores }\end{array}$ & Infusión & Oral & $\begin{array}{l}\text { Acción carminativa, } \\
\text { espasmolítico, antidiarreica, } \\
\text { digestiva, sedante y } \\
\text { enfermedades de la piel. }\end{array}$ & 37 & 0.39 \\
\hline 7 & ASTERACEAE & $\begin{array}{l}\text { Taraxacum } \\
\text { campylodes } \\
\text { G.E. Haglund. }\end{array}$ & $\begin{array}{l}\text { "diente de } \\
\text { león" }\end{array}$ & Hoja & Cocimiento & Oral & $\begin{array}{l}\text { Afecciones biliares, de la } \\
\text { vesícula, riñón, afecciones } \\
\text { hepáticas, antidiarreico y } \\
\text { depuración de la sangre. }\end{array}$ & 12 & 0.13 \\
\hline 8 & ASTERACEAE & $\begin{array}{l}\text { Cichorium } \\
\text { intybus L. }\end{array}$ & $\begin{array}{l}\text { "achicoria", } \\
\text { "amargón" }\end{array}$ & Hoja & Cocimiento & Oral & $\begin{array}{l}\text { Depuración de la sangre, } \\
\text { mineralizante, laxante, } \\
\text { contra trastornos hepáticos e } \\
\text { inflamaciones renales. }\end{array}$ & 5 & 0.05 \\
\hline 9 & ASTERACEAE & $\begin{array}{l}\text { Ambrosia } \\
\text { peruviana } \\
\text { Willd. }\end{array}$ & $\begin{array}{l}\text { "marco", } \\
\text { "altamisa" }\end{array}$ & $\begin{array}{l}\text { Tallo y } \\
\text { hojas }\end{array}$ & Cocimiento & Oral & $\begin{array}{l}\text { Contra la tos, disentería, } \\
\text { alivia los bronquios y } \\
\text { antinflamatorio. }\end{array}$ & 4 & 0.04 \\
\hline 10 & ASTERACEAE & $\begin{array}{l}\text { Flaveria } \\
\text { bidentis L. }\end{array}$ & $\begin{array}{l}\text { "mata } \\
\text { gusano" }\end{array}$ & $\begin{array}{l}\text { Toda la } \\
\text { planta }\end{array}$ & $\begin{array}{l}\text { Infusión y } \\
\text { cocimiento }\end{array}$ & Oral & $\begin{array}{l}\text { Acción anti disentérica, } \\
\text { antitusigena, amigdalitis, } \\
\text { úlceras dérmicas (heridas). }\end{array}$ & 3 & 0.03 \\
\hline 11 & BRASSICACEAE & $\begin{array}{l}\text { Lepidium } \\
\text { meyenii Walp. }\end{array}$ & "maca" & $\begin{array}{l}\text { Raíz } \\
\text { engrosa } \\
\text { da }\end{array}$ & Cocimiento & Oral & $\begin{array}{l}\text { Aumenta la fertilidad, } \\
\text { vitalidad sexual, la } \\
\text { capacidad mental y refuerza } \\
\text { el sistema inmunológico. }\end{array}$ & 36 & 0.38 \\
\hline 12 & BROMELIACEAE & $\begin{array}{l}\text { Ananas } \\
\text { comosus L. }\end{array}$ & "piña" & $\begin{array}{l}\text { Infrutes } \\
\text { cencia }\end{array}$ & $\begin{array}{l}\text { Jugo y } \\
\text { cocimiento }\end{array}$ & Oral & $\begin{array}{l}\text { Acción antiinflamatoria, } \\
\text { anticoagulante, contra } \\
\text { mareos, problemas del } \\
\text { hígado e ictericia. }\end{array}$ & 31 & 0.32 \\
\hline 13 & CACTACEAE & $\begin{array}{l}\text { Opuntia ficus- } \\
\text { indica (L.) } \\
\text { Mill. }\end{array}$ & $\begin{array}{l}\text { "tuna", } \\
\text { "cacto", } \\
\text { "pupa" }\end{array}$ & $\begin{array}{l}\text { Cladodi } \\
0\end{array}$ & Extracto & Oral & $\begin{array}{l}\text { Ablanda la tos, } \\
\text { antidiabética, diurética, } \\
\text { laxante, desparasita y } \\
\text { apresura el parto. }\end{array}$ & 14 & 0.15 \\
\hline 14 & CAPRIFOLIACEAE & $\begin{array}{l}\text { Sambucus } \\
\text { peruviana } \\
\text { Kunth }\end{array}$ & "saúco," & $\begin{array}{l}\text { Toda la } \\
\text { planta }\end{array}$ & $\begin{array}{l}\text { Infusión y } \\
\text { cocimiento }\end{array}$ & Oral & $\begin{array}{l}\text { Contra inflamación de la } \\
\text { vejiga, próstata, antiséptico, } \\
\text { depurativos, tos y asma }\end{array}$ & 6 & 0.06 \\
\hline 15 & CARICACEAE & $\begin{array}{l}\text { Carica } \\
\text { papaya } \mathrm{L} .\end{array}$ & $\begin{array}{l}\text { "papaya", } \\
\text { "papayo" }\end{array}$ & $\begin{array}{l}\text { Fruto y } \\
\text { semillas }\end{array}$ & $\begin{array}{l}\text { Jugo y } \\
\text { cocimiento }\end{array}$ & Oral & $\begin{array}{l}\text { Combatir la dispepsia, } \\
\text { gastroenteritis, diarrea } \\
\text { infantil, úlceras, indigestión } \\
\text { y contra parásitos } \\
\text { intestinales. }\end{array}$ & 22 & 0.23 \\
\hline 16 & ERICACEAE & $\begin{array}{l}\text { Vaccinium } \\
\text { myrtillus } \mathrm{L} \text {. }\end{array}$ & "arándano" & Fruto & jugo & Oral & $\begin{array}{l}\text { Propiedades antisépticas, } \\
\text { astringentes y antidiarreicas. }\end{array}$ & 27 & 0.28 \\
\hline 17 & EQUISETACEAE & $\begin{array}{l}\text { Equisetum } \\
\text { bogotense } \\
\text { Kunth.. }\end{array}$ & $\begin{array}{l}\text { "cola de } \\
\text { caballo" }\end{array}$ & $\begin{array}{l}\text { Toda la } \\
\text { planta }\end{array}$ & $\begin{array}{l}\text { Infusión y } \\
\text { cocimiento }\end{array}$ & Oral & $\begin{array}{l}\text { Acción antidiarreica, } \\
\text { digestiva, depurativa y } \\
\text { desinflamante. }\end{array}$ & 20 & 0.21 \\
\hline 18 & EUPHORBIACEAE & $\begin{array}{l}\text { Manihot } \\
\text { esculenta } \\
\text { Crantz. }\end{array}$ & "yuca" & Raiz & Cocimiento & Oral & $\begin{array}{l}\text { Acción antiinflamatoria, } \\
\text { inmunoestimulante, } \\
\text { desintoxicante y depurativa. }\end{array}$ & 34 & 0.35 \\
\hline 19 & EUPHORBIACEAE & $\begin{array}{l}\text { Phyllanthus } \\
\text { niruri L. }\end{array}$ & "chancapiedra" & $\begin{array}{l}\text { Tallo y } \\
\text { hojas }\end{array}$ & Cocimiento & Oral & $\begin{array}{l}\text { Como diurético, disolver } \\
\text { cálculos biliares, renales y } \\
\text { del hígado, hepatitis y } \\
\text { antipirético. }\end{array}$ & 12 & 0.13 \\
\hline 20 & FABACEAE & $\begin{array}{l}\text { Tamarindus } \\
\text { indica } \mathrm{L} \text {. }\end{array}$ & "tamarindo" & Fruto & Jugo & Oral & $\begin{array}{l}\text { Propiedad antibacteriana, } \\
\text { anti fúngica, contra } \\
\text { afecciones biliares, del bazo, } \\
\text { vesícula biliar, } \\
\text { estreñimiento, inflamación y } \\
\text { laxante. }\end{array}$ & 14 & 0.15 \\
\hline 21 & FABACEAE & $\begin{array}{l}\text { Medicago } \\
\text { sativa } \mathrm{L} \text {. }\end{array}$ & "alfalfa" & $\begin{array}{l}\text { Tallo y } \\
\text { hojas }\end{array}$ & Extracto & Oral & $\begin{array}{l}\text { Propiedades anti anémicas, } \\
\text { antihemorrágicas y } \\
\text { recalcificantes. }\end{array}$ & 31 & 0.32 \\
\hline
\end{tabular}




\begin{tabular}{|c|c|c|c|c|c|c|c|c|c|}
\hline 22 & FABACEAE & $\begin{array}{l}\text { Desmodium } \\
\text { molliculum } \\
\text { (Kunth) DC. }\end{array}$ & $\begin{array}{l}\text { "pie de } \\
\text { perro" }\end{array}$ & $\begin{array}{l}\text { Toda la } \\
\text { planta }\end{array}$ & Infusión & Oral & $\begin{array}{l}\text { Acción diurética, calmante y } \\
\text { para curar enfermedades } \\
\text { hepáticas. }\end{array}$ & 29 & 0.30 \\
\hline 23 & LAMIACEAE & $\begin{array}{l}\text { Origanum } \\
\text { vulgare } \mathrm{L} .\end{array}$ & "orégano" & $\begin{array}{l}\text { Tallo y } \\
\text { hojas }\end{array}$ & $\begin{array}{l}\text { Infusión y } \\
\text { condimento }\end{array}$ & Oral & $\begin{array}{l}\text { Alivia los sintomas del } \\
\text { resfriado, el asma, sedante, } \\
\text { sistema nervioso y útero. }\end{array}$ & 15 & 0.16 \\
\hline 24 & LAMIACEAE & $\begin{array}{l}\text { Thymus } \\
\text { vulgaris L. }\end{array}$ & "tomillo" & Fruto & Fresco & Oral & $\begin{array}{l}\text { Reducir la tensión arterial, } \\
\text { mejorar la higiene } \\
\text { bucodental, la gingivitis y } \\
\text { problemas de encías. }\end{array}$ & 4 & 0.04 \\
\hline 25 & LAMIACEAE & $\begin{array}{l}\text { Mentha } \\
\text { spicata L. }\end{array}$ & $\begin{array}{l}\text { "hierba } \\
\text { buena" }\end{array}$ & $\begin{array}{l}\text { Tallo y } \\
\text { hojas }\end{array}$ & Fresco & Oral & $\begin{array}{l}\text { Contra el nerviosismo, } \\
\text { insomnios, calambres, tos y } \\
\text { dolores espasmódicos. }\end{array}$ & 36 & 0.38 \\
\hline 26 & LAMIACEAE & $\begin{array}{l}\text { Mentha } \times \\
\text { piperita L. }\end{array}$ & "menta" & $\begin{array}{l}\text { Toda la } \\
\text { planta }\end{array}$ & Infusión & Oral & $\begin{array}{l}\text { Usada como estimulante, } \\
\text { carminativa, colagoga y } \\
\text { espasmolitico. }\end{array}$ & 16 & 0.17 \\
\hline 27 & LAURACEAE & $\begin{array}{l}\text { Cinnamomum } \\
\text { verum J.Presl. }\end{array}$ & "canela" & Corteza & Infusión & Oral & $\begin{array}{l}\text { Tratamientos de resfriados, } \\
\text { dolor de garganta, fiebre y } \\
\text { vómitos. }\end{array}$ & 9 & 0.09 \\
\hline 28 & LILIACEAE & Allium cepa $\mathrm{L}$. & "cebolla" & Raiz & $\begin{array}{l}\text { Fresco y } \\
\text { macerado }\end{array}$ & Oral & $\begin{array}{l}\text { Acción antiséptica, } \\
\text { diurética, expectorante, } \\
\text { desintoxicante, digestiva y } \\
\text { regeneración de la sangre } \\
\text { (anemia). }\end{array}$ & 16 & 0.17 \\
\hline 29 & LILIACEAE & $\begin{array}{l}\text { Allium } \\
\text { sativum L. }\end{array}$ & "ajo" & Bulbo & Extracto & Oral & $\begin{array}{l}\text { Acción antiespasmódico, } \\
\text { vasodilatador, expectorante, } \\
\text { diurético y estimulante en el } \\
\text { sistema nervioso. }\end{array}$ & 21 & 0.22 \\
\hline 30 & LILIACEAE & $\begin{array}{l}\text { Aloe vera }(\mathrm{L} .) \\
\text { Burm.f. }\end{array}$ & "sábila" & Hojas & $\begin{array}{l}\text { Extracto y } \\
\text { emplasto }\end{array}$ & $\begin{array}{l}\text { Oraly } \\
\text { tópica }\end{array}$ & $\begin{array}{l}\text { Acción } \\
\text { antiasmática,desinflamante, } \\
\text { efecto cicatrizante, laxante. }\end{array}$ & 40 & 0.42 \\
\hline 31 & LINACEAE & $\begin{array}{l}\text { Linum } \\
\text { usitatissimum } \\
\text { L. }\end{array}$ & "linaza" & Semilla & Cocimiento & Oral & $\begin{array}{l}\text { Propiedades laxantes, } \\
\text { menstruación, náuseas, } \\
\text { cálculos biliares y } \\
\text { enfermedades respiratorias. }\end{array}$ & 44 & 0.46 \\
\hline 32 & MYRTACEAE & $\begin{array}{l}\text { Eucalyptus } \\
\text { globulus } \\
\text { Labill. }\end{array}$ & "eucalipto" & Hojas & $\begin{array}{l}\text { Infusión y } \\
\text { cocimiento }\end{array}$ & $\begin{array}{l}\text { Oral y } \\
\text { baños }\end{array}$ & $\begin{array}{l}\text { Acción antiséptica, } \\
\text { depurativa, desinflamante, } \\
\text { enfermedades de las vías } \\
\text { respiratorias y cicatrizante. }\end{array}$ & 14 & 0.15 \\
\hline 33 & PAPAVERACEAE & $\begin{array}{l}\text { Argemone } \\
\text { subfusiformis } \\
\text { Ownbey. }\end{array}$ & $\begin{array}{l}\text { "cardosanto } \\
\text { " }\end{array}$ & Semilla & Cocimiento & Oral & $\begin{array}{l}\text { Acción sedante, purgante, } \\
\text { narcóticas e hipnóticas. }\end{array}$ & 4 & 0.04 \\
\hline 34 & PASSIFLORACEAE & $\begin{array}{l}\text { Passiflora } \\
\text { edulis Sims. }\end{array}$ & "maracuyá" & Fruto & Jugo & Oral & $\begin{array}{l}\text { Disminuye la presión } \\
\text { arterial, hipotensora, } \\
\text { insomnio y tétano. }\end{array}$ & 26 & 0.27 \\
\hline 35 & PIPERACEAE & $\begin{array}{l}\text { Piper auritum } \\
\text { Kunth. }\end{array}$ & $\begin{array}{l}\text { "hierba } \\
\text { santa" }\end{array}$ & Hojas & $\begin{array}{l}\text { Emplasto y } \\
\text { macerado }\end{array}$ & Oral & $\begin{array}{l}\text { Acción para problemas } \\
\text { respiratorios, disminuye el } \\
\text { dolor de cabeza y disminuye } \\
\text { la inflamación de la matriz } \\
\text { después del parto. }\end{array}$ & 11 & 0.11 \\
\hline 36 & PIPERACEAE & $\begin{array}{l}\text { Piper } \\
\text { aduncum } \mathrm{L} \text {. }\end{array}$ & "matico" & Hojas & $\begin{array}{l}\text { Infusión y } \\
\text { cocimiento }\end{array}$ & Oral & $\begin{array}{l}\text { Acción desinflamante, } \\
\text { sedante y anticancerígeno. }\end{array}$ & 31 & 0.32 \\
\hline 37 & PLANTAGINACEAE & $\begin{array}{l}\text { Plantago } \\
\text { major L. }\end{array}$ & "llantén" & Hojas & $\begin{array}{l}\text { Infusión, } \\
\text { cocimiento } \\
\text { y emplasto }\end{array}$ & $\begin{array}{l}\text { Oral y } \\
\text { tópica }\end{array}$ & $\begin{array}{l}\text { Afecciones hepáticas, } \\
\text { pulmones, amigdalitis, } \\
\text { antidiarreico, cálculos } \\
\text { biliares, úlceras } \\
\text { estomacales, sarna y } \\
\text { regenera la flora intestinal. }\end{array}$ & 14 & 0.15 \\
\hline
\end{tabular}




\begin{tabular}{|c|c|c|c|c|c|c|c|c|c|}
\hline 38 & POACEAE & $\begin{array}{l}\text { Hordeum } \\
\text { vulgare L. }\end{array}$ & "cebada" & Semilla & $\begin{array}{l}\text { Infusión y } \\
\text { cocimiento }\end{array}$ & Oral & $\begin{array}{l}\text { Antidiarreico, diurético, } \\
\text { hipotensión y sedante. }\end{array}$ & 38 & 0.40 \\
\hline 39 & POACEAE & $\begin{array}{l}\text { Cymbopogon } \\
\text { citratus (DC.) } \\
\text { Stapf. }\end{array}$ & $\begin{array}{l}\text { "hierba } \\
\text { luisa" }\end{array}$ & Hojas & $\begin{array}{l}\text { Infusión y } \\
\text { cocimiento }\end{array}$ & Oral & $\begin{array}{l}\text { Antiinflamatorio de las vías } \\
\text { urinarias, antipirético, } \\
\text { antiespasmódico, analgésico } \\
\text { y digestivo. }\end{array}$ & 44 & 0.46 \\
\hline 40 & RUBIACEAE & $\begin{array}{l}\text { Morinda } \\
\text { citrifolia L. }\end{array}$ & "noni" & Fruto & Extracto & Oral & $\begin{array}{l}\text { Mantenimiento de la salud } \\
\text { cardiovascular, protección } \\
\text { del hígado y la cicatrización } \\
\text { de heridas. }\end{array}$ & 38 & 0.40 \\
\hline 41 & RUBIACEAE & $\begin{array}{l}\text { Uncaria } \\
\text { tomentosa } \\
\text { (Willd. ex } \\
\text { Schult.) DC. }\end{array}$ & $\begin{array}{l}\text { "uña de } \\
\text { gato" }\end{array}$ & Corteza & Cocimiento & Oral & $\begin{array}{l}\text { Activa el sistema } \\
\text { inmunológico, antiartrítico, } \\
\text { anticancerígeno, } \\
\text { antiinflamatorio y } \\
\text { antioxidante. }\end{array}$ & 17 & 0.18 \\
\hline 42 & RUTACEAE & $\begin{array}{l}\text { Ruta } \\
\text { graveolens L. }\end{array}$ & "ruda" & $\begin{array}{l}\text { Toda la } \\
\text { planta }\end{array}$ & Cocimiento & baños & $\begin{array}{l}\text { Calmar dolor en golpes, } \\
\text { distenciones e inflamaciones } \\
\text { en las enfermedades } \\
\text { reumáticas. }\end{array}$ & 12 & 0.13 \\
\hline 43 & RUTACEAE & $\begin{array}{l}\text { Citrus } x \\
\text { sinensis Pers. }\end{array}$ & "naranja" & Fruto & Jugo & Oral & $\begin{array}{l}\text { Contra afecciones } \\
\text { digestivas, respiratorias, } \\
\text { nerviosas, cardiacas y } \\
\text { tópicas. }\end{array}$ & 25 & 0.26 \\
\hline 44 & RUTACEAE & $\begin{array}{l}\text { Citrus limon } \\
\text { (L.) Osbeck. }\end{array}$ & "limón" & Fruto & Jugo & Oral & $\begin{array}{l}\text { Contra afecciones } \\
\text { digestivas, respiratorias, } \\
\text { nerviosas, cardiacas, tópicas } \\
\text { (cicatrizante). }\end{array}$ & 41 & 0.43 \\
\hline 45 & SOLANACEAE & $\begin{array}{l}\text { Solanum } \\
\text { tuberosum L. }\end{array}$ & "papa" & $\begin{array}{l}\text { Tubércu } \\
\text { lo }\end{array}$ & $\begin{array}{l}\text { Cocimiento } \\
\text {, emplasto } \\
\text { y extracto }\end{array}$ & $\begin{array}{l}\text { Oral y } \\
\text { tópica }\end{array}$ & $\begin{array}{l}\text { Propiedades desinflamantes, } \\
\text { calmantes, analgésicas y } \\
\text { antiácido. }\end{array}$ & 39 & 0.41 \\
\hline 46 & TILIACEAE & $\begin{array}{l}\text { Tilia } \\
\text { platyphyllos } \\
\text { Scop. }\end{array}$ & "tilo" & Hojas & Infusión & Oral & $\begin{array}{l}\text { Activa el sistema } \\
\text { inmunológico, afecciones } \\
\text { pulmonares, gripe, estrés y } \\
\text { sedante. }\end{array}$ & 22 & 0.23 \\
\hline 47 & TROPAEOLACEAE & $\begin{array}{l}\text { Tropaeolum } \\
\text { majus L. }\end{array}$ & "mastuerzo" & Flores & Fresco & Oral & $\begin{array}{l}\text { Acción expectorante, } \\
\text { fluidificante de las } \\
\text { secreciones bronquiales y } \\
\text { antibiótico. }\end{array}$ & 5 & 0.05 \\
\hline 48 & URTICACEAE & $\begin{array}{l}\text { Urtica dioica } \\
\mathrm{L} \text {. }\end{array}$ & "ortiga" & Hojas & Cocimiento & $\begin{array}{l}\text { Oral, } \\
\text { Tópica }\end{array}$ & $\begin{array}{l}\text { Acción depurativa de la } \\
\text { sangre, mejora la } \\
\text { circulación, antidiabética y } \\
\text { antihemorroidal. }\end{array}$ & 5 & 0.05 \\
\hline 49 & VALERIANACEAE & $\begin{array}{l}\text { Valeriana } \\
\text { officinalis } \mathrm{L} \text {. }\end{array}$ & "valeriana" & Raíz & Infusión & Oral & $\begin{array}{l}\text { Antiasmática, debilidad, } \\
\text { dolor de cabeza, sistema } \\
\text { nervioso y estrés. }\end{array}$ & 9 & 0.09 \\
\hline 50 & ZINGEBERACEAE & $\begin{array}{l}\text { Zingiber } \\
\text { officinale } \\
\text { Roscoe. }\end{array}$ & "kión" & $\begin{array}{l}\text { Rizoma } \\
\mathrm{s}\end{array}$ & $\begin{array}{l}\text { Extracto y } \\
\text { cocimiento }\end{array}$ & Oral & $\begin{array}{l}\text { Antiasmática, antidiarreico, } \\
\text { antiséptica, diurética, } \\
\text { resfríos y úlceras } \\
\text { estomacales. }\end{array}$ & 35 & 0.36 \\
\hline
\end{tabular}

Leyenda: P.U $=$ Parte utilizada, T.P $=$ tipo de preparación, $F . A=$ forma de administración, R.U= Reporte de uso, IVU= Índice de valor de uso. 


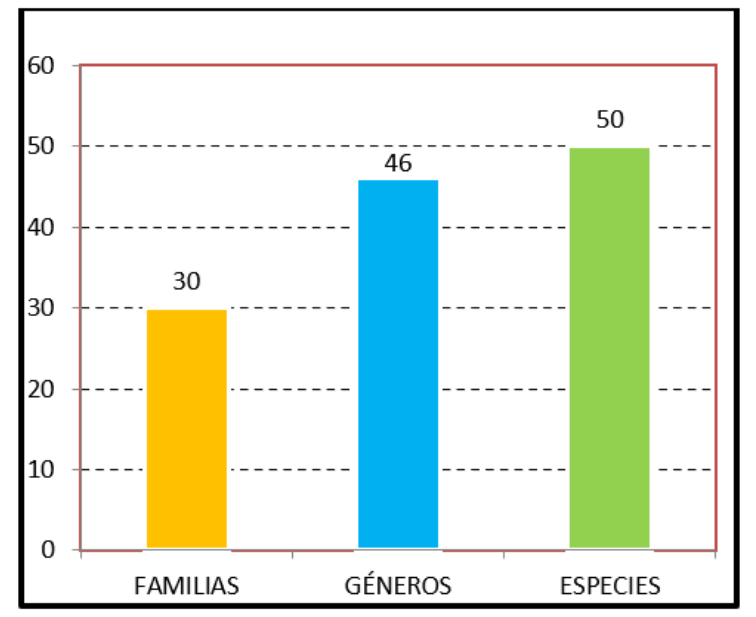

Figura 1. Distribución en Familias, Géneros y Especies de la flora empleada por el poblador de Víctor Larco, Trujillo, La Libertad, Perú

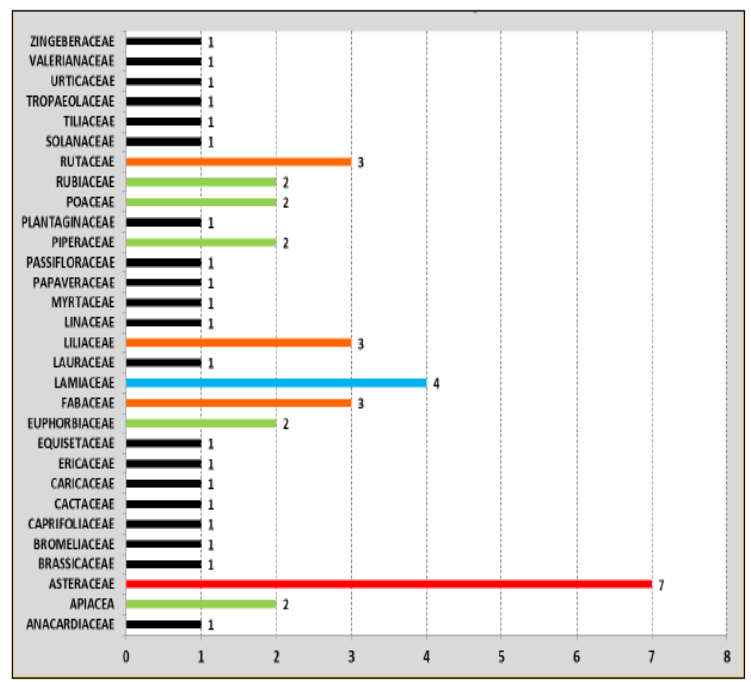

Figura 2. Número de especies por familia de la flora empleada por el poblador de Víctor Larco, Trujillo, La Libertad, Perú.

La presente investigación reporta un total de 50 especies de plantas empleadas desde tiempos inmemorables, distribuidas en 46 géneros y 30 Familias (tabla 1, Figura 1), que de alguna $\mathrm{u}$ otra forma representa una herencia para el poblador peruano en general, y del distrito de Víctor Larco en particular, lo que concuerda con Tovar (2001), quien afirma que el antiguo poblador peruano desde tiempos muy remotos conocía el correcto y eficaz uso de las plantas medicinales y su influencia en la mejora de la calidad de vida. Pudiéndose observar a su vez que las especies más utilizada y por consiguiente más importantes para el poblador del distrito de Víctor Larco en el tratamiento de sus enfermedades y/o dolencias, según su índice de valor de uso (IVU) son : Linum usitatissimum L. "linaza" (IVU=0.46), Cymbopogon citratus (DC.) Stapf "hierba luisa” (IVU=0.46), Citrus limon (L.) Osbeck "limón" (IVU=0.43), Aloe vera (L.) Burm.f. "sábila" (IVU=0.42), Solanum tuberosum L. "papa" (IVU=0.41), Hordeum vulgare L. "cebada" (IVU=0.40) y Morinda citrifolia L. "noni" (IVU=0.40).

Cabe destacar, que la especie Linum usitatissimum L. "linaza", posee un contenido en alto grado del ácido graso poliinsaturado alfa-linoléico (Omega-3), que representa en su composición 50 - 55\% de los ácidos grasos totales, y de $40 \%$ de fibras de su peso total, siendo el 10\% soluble y el 30\% insoluble, además de las proteínas, ligninas, vitaminas y minerales; sustancias que le confieren a esta especie, disminuir el riesgo del desarrollo de las enfermedades cardiovasculares, cáncer, actividad anti-inflamatoria, efecto laxante y antioxidante, además de la prevención de síntomas de la menopausia (Mostacero et al., 2011). De igual manera la especie Cymbopogon citratus (DC.) Stapf "hierba luisa" en medicina tradicional, es consumida en decocción o infusión como anticatarral, antiespasmódico, hipotensor, antipirético y tranquilizante (Mostacero et al., 2011).

Por otro lado, el hecho de que las familias: Asteraceae, Lamiaceae, Rutaceae, Liliaceae y Fabaceae; sean las más representativas por el número de especies (Figura 3); avala lo descrito por Zielinska y Michalska (2016) y Castillo-Vera et al. (2017), quienes afirman que las Asteraceae y Lamiaceae, principalmente, presentan un amplio rango de distribución y 
un cuantioso número de metabolitos secundarios, capaces de aliviar y/o curar un sin número de enfermedades relacionadas a los sistemas digestivo, respiratorio y genito- urinario por los que son las más empleadas en medicina tradicional; de allí que en esta investigación, las personas del distrito de Victor Larco atribuyen a estas familias la capacidad de curar y/o aliviar distintas afecciones, como las hepáticas, de la vejiga; así como también se les confiere un efecto antidiarreico, antiespasmódica, carminativa, depurativa, disentería, enfermedades de la piel, inflamación, úlceras estomacales, entre otros malestares y/o enfermedades (Mostacero et al., 2011).

Finalmente, es importante resaltar, que en cuanto al porcentaje de utilidad, referente a la parte empleada del vegetal (tabla 1); destaca la preferencia a la hoja en un $24 \%$ debido a su fácil manipulación, reelección, reconocimiento y preparación; mientras que un $14 \%$ tiene preferencia al empleo de toda la planta así como a los frutos, debido a su mayor rango de material y propiedades como vitaminas, sales minerales y antioxidantes, que se puede encontrar, aumentando de esta manera la eficiencia en el tratamiento y/o cura de enfermedades, lo que concuerda con lo descrito por (Muñoz, 1996). Por todo lo descrito en los párrafos anteriores, se espera que la presente investigación, sirva de base para posteriores estudios del tipo aplicado; toda vez que es imprescindible establecer nuevas líneas de investigación en nuestro país, relacionadas al aprovechamiento sustentable de nuestros recursos; a la par que se rescata el legado ancestral etnobotánico heredado de nuestros antepasados.

\section{CONCLUSIONES}

El poblador del distrito de Víctor Larco; emplea 50 especies de plantas medicinales, distribuidas en 46 géneros y 30 familias, de las que destacan por el número de especies a las: Asteraceae (7), Lamiaceae (4), Rutaceae (3), Liliaceae (3) y Fabaceae (3). Las especies más importantes empleadas en el tratamiento y/o cura de las enfermedades padecidas por el poblador del distrito de Victor Larco, según su Indice de Valor de Uso (IVU) son: Linum usitatissimum L. "linaza", Cymbopogon citratus (DC.) Stapf "hierba luisa", Citrus limon (L.) Osbeck "limón”, Aloe vera (L.) Burm.f. "sábila", Solanum tuberosum L. "papa", Hordeum vulgare L. "cebada" y Morinda citrifolia L. "noni".

\section{REFERENCIAS BIBLIOGRÁFICAS}

Bailey, K. (1994). Methods of Social Research. Ed. The Free Press, New York, USA.

Bocanegra, L.; Bocanegra, F.; Mostacero, J. (2011). Efectividad de la medicina herbolaria y su impacto en la calidad de vida de los pobladores de Curgos, Perú. UCV-Scientia, 3(1):23-34.

Bhat, R.; Paliyath, G. (2016). Fruits of Tropical Climates: Dietary Importance and Health Benefits. Reference Module in Food Science, from Encyclopedia of Food and Health: 144-149.

Boeing, H.; Bechthold, A.; Bub, A.; Ellinger, S.; Haller, D.; Kroke, A.; Leschik-Bonnet, E.; Müller, M.; Oberritter, H.; Schulze, M.; Stehle, P. \& Watzl, B. (2012). Critical review: vegetables and fruit in the prevention of chronic diseases. Eur J Nutr 51(6): 637-663.

Brack, A. (2000). Biodiversidad y biocomercio en el Perú. Informe para CONAM y UNTAD. 100 pág.

Brako, L.; Zarucchi, J. (1993). Catálogo de las Angiospermas y Gimnospermas del Perú. 
Monogr. Syst. Bot. Missouri Bot. Garden. Vol 45 .

Cárdenas, G.; Arrazola, G.; Villalba, M. (2015). Frutas tropicales: fuente de compuestos bioactivos naturales en la industria de alimentos. Ingenium 17(33): 29-40.

Castillo-Vera, H.; Cochachin, E.; Albán, J. (2017). Plantas comercializadas por herbolarios en el mercado del distrito de Cajabamba (Cajamarca, Perú). Blacpma, 16 (3): 303-318.

De La Cruz, J; Mostacero, J.(2019). Uso de plantas medicinales para la cura de enfermedades y/o dolencias: El caso del poblador de la provincia de Trujillo, Perú. Manglar 16(2): 119-124

Freedman, D.; Peña, N.; Friedman, D.; Ory, M.; Flocke, S.; Barni, M. \& Hébert, J. (2014). Extending Cancer Prevention to Improve Fruit and Vegetable Consumption. J Cancer Educ. 29(4): 790-795.

Goszcz, K.; Deakin, S.; Duthie, G.; Stewart, D.; Leslie, S.; Megson, I. (2015). Antioxidants in cardiovascular therapy: panacea or false hope. Front. Cardiovasc. Med 2: 29.

Instituto Nacional de Estadística e Informática (INEI) (2018). Censos Nacionales de Población y Vivienda.

Mittermeier, R.; Mast, R.; Del Prado, C.; Mittermeier, C. (1997). Peru Megadiversity: Earth's Biologically Wealthiest Nations. Monterrey, México: CEMEX. 282-297.\}

Mostacero, J.; Mejía, F.; Zelada, W.; Medina, C. (2007). Biogeografía del Perú. Asamblea Nacional de Rectores. Trujillo - Perú.
Mostacero, J.; Mejía, F.; Gamarra, O. (2009). Fanerógamas del Perú: Taxonomía, utilidad y ecogeografía. CONCYTEC. Trujillo - Perú.

Mostacero, J.; Castillo, F.; Mejía, F.; Gamarra, O.; Charcape, J.; Ramírez, R. (2011). Plantas Medicinales del Perú: Taxonomía, Ecogeografía, Fenología y Etnobotánica. Trujillo - Perú: AsambleaNacionaldeRectoresFondoEditorial.

Mostacero, J.; Peláez, F.; Alarcón, N.; De La Cruz, A.; Alva, R.; Charcape, M. (2019). Plantas utilizadas para el tratamiento del cáncer expendidas en los principales mercados de la provincia de Trujillo, Perú, 2016-2017. Bol Latinoam Caribe PlantMedAromat, 18(1): 81-94.

Muñoz, F. (1996) Plantas medicinales en España: estudio, cultivo y procesado. $1^{\mathrm{a}} \mathrm{Ed}, 2^{\mathrm{a}}$ reimp. Madrid: Mundi-Prensa; 22-24.

Rengifo, E.; Rios, S.; Fachín, L.; Vargas, G. (2017). Saberes ancestrales sobre el uso de flora y fauna en la comunidad indígena Tikuna de Cushillo Cocha, zona fronteriza Perú-Colombia-Brasil. Revista peruana de biología, 24(1): 67-78.

Rodríguez, Y.; Valdés, M.; Hernández, H.; Soria, S. (2019.) Guía metodológica para estudio etnobotánico de especies forestales en comunidades amazónicas y afines. Rev cubana ciencias forestales 7 (1): 97-110.

Tovar, O., (2001) Plantas medicinales del Valle del Mantaro. Consejo Nacional de Ciencia y Tecnología (Peru). CONCYTEC.

Zambrano, L.; Buenaño, M.; Mancera, N.; Jiménez, E. (2015). Estudio etnobotánico de plantas medicinales utilizadas por los habitan- 
tes del área rural de la Parroquia San Carlos, Quevedo, Ecuador. Rev.Univ. Salud 17:97- 111.

Zielinska, M.; Michalska, A. (2016). Microwave-assisted drying of blueberry (Vaccinium corymbosum L.) fruits: Drying kinetics, polyphenols, anthocyanins, antioxidant capacity, colour and texture. Food Chemistry 212(1): 671-680. 